\title{
Our perception of dental comp
do we have the right mindset?
}

\author{
By Sidra Ahmed Suleman, Foundation Dentist, Leicester
}

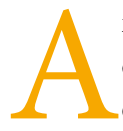
ny expression of patient dissatisfaction can be disheartening, especially for a young dentist who is still at an early stage of growth and development. Will I get a complaint? Will someone take legal action against me? These are all valid concerns most foundation dentists have as they embark on their training. As we are fairly sheltered from such issues during dental school it can be particularly hard to experience these situations for the first time.

We all are aware that complaints are inevitable as we cannot please all our patients. Yet there still seems to be hesitation towards discussing and sharing our experiences with one another because of the plays a huge role in maintaining the stigma that continues to exist.

As a foundation dentist, managing complaints can be much harder in some ways as we are still developing our skill and self-confidence. Equally so, it is important to remember that getting a complaint does not necessarily mean you are at fault or incompetent, although it is hard not to feel that way sometimes. These days there is a greater focus on reflection and feedback and complaints offer an invaluable learning experience which is still underappreciated.

A question to consider is are we just assuming our patients feel comfortable enough to make a complaint? How are we actively assessing and ensuring this is the

\section{'It is generally much easier to discuss a clinical case with a colleague than it is to discuss a complaint or legal concern.'}

stigma attached to it. However, could this be down to the mindset and culture we have created ourselves? With dentistry becoming such a competitive market is it feasible to talk about our struggles with others and online? In order to explore these issues, we need to understand and question why things have become this way.

As soon as we hear the word 'complaint' most people will associate negative connotations with it. Although experiencing a complaint can be difficult and stressful, it is important not to label it as a 'negative situation' in our minds. Doing this can affect the way we approach and feel about the complaint and potentially change the outcome. So, it is important to restructure the way we perceive dental complaints as this case? The reality is that not many systems are in place for this. We mainly depend on patients to come to us with a concern which is not the ideal approach. As clinicians we should be asking all our patients how they feel about the care they are receiving. A comment box is commonly used but again, we are relying on patients for it to be of any use. Therefore, we should make such conversations part of our daily routine. We are not only trying to address concerns but also prevent patients from developing resentment over issues that they would not otherwise talk about.

With regards to handling complaints we are generally taught to resolve them as soon as possible and I appreciate that this is important as delaying things could make the

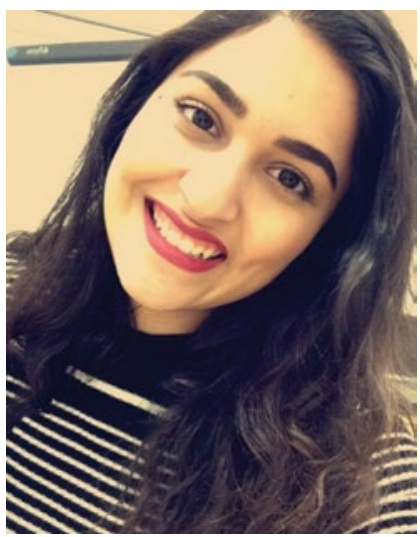

situation worse. However, on the contrary I do feel this mindset of 'fix the problem quickly' can sometimes distract from the true purpose of understanding and helping the patient in a way that is best for them and not just for us. From my own experience I have learned that sometimes patients' upset may stem from other issues they are facing. Therefore, it is equally as important to understand why the complaint has developed in the first place. This can be difficult to do especially when you may feel 'attacked' and time pressured at the time, however, this is when putting the patients' best interests first truly comes into play.

The truth of the matter is that it is generally much easier to discuss a clinical case with a colleague than it is to discuss a complaint or legal concern. I feel a main reason for this is fear of judgement. Although we make constant efforts to avoid making assumptions about our patients, unfortunately the same behaviour is not always reserved for our colleagues as well. This strengthens the stigma around complaints and makes it harder for us to learn and seek help from each other. With dentistry being such a demanding and stressful career, it is not surprising that many dentists struggle with their mental health at some point. Complaints are just one of the many challenging things we all deal with and so it is in our interest to establish a culture which is non-judgemental, supportive and patient focused. Young dentists have a vital role in changing and influencing key issues we face within our community and it is essential we use our voice to achieve this. 